\title{
Evidence for rotationally driven plasma transport in Saturn's magnetosphere
}

\author{
T. W. Hill, ${ }^{1}$ A. M. Rymer, ${ }^{2}$ J. L. Burch, ${ }^{3}$ F. J. Crary, ${ }^{3}$ D. T. Young, ${ }^{3}$ M. F. Thomsen, ${ }^{4}$ \\ D. Delapp, ${ }^{4}$ N. André, ${ }^{5}$ A. J. Coates, ${ }^{2}$ and G. R. Lewis ${ }^{2}$
}

Received 3 February 2005; revised 20 March 2005; accepted 13 April 2005; published 22 June 2005.

[1] Radial convective transport of plasma in a rotationdominated magnetosphere implies alternating longitudinal sectors of cooler, denser plasma moving outward and hotter, more tenuous plasma moving inward. The Cassini Plasma Spectrometer (CAPS) has provided dramatic new evidence of this process operating in the magnetosphere of Saturn. The inward transport of hot plasma is accompanied by adiabatic gradient and curvature drift, producing a V-shaped dispersion signature on a linear energy-time plot. Of the many $(\sim 100)$ such signatures evident during the first two Cassini orbits, we analyze a subset (48) that are sufficiently isolated to allow determination of their ages, widths, and injection locations. Ages are typically $<10.8 \mathrm{hr}$ (Saturn's rotation period) but range up to several rotation periods. Widths are typically $<1 R_{S}$ (Saturn's radius) but range up to several $R_{S}$. Injection locations are randomly distributed in local time and in Saturnian longitude. The apex of the V sometimes coincides with a localized density cavity in the cooler background plasma, and usually coincides with a localized diamagnetic depression of the magnetic field strength. These signatures are fully consistent with the convective motions that are expected to result from the centrifugal interchange instability. Citation: Hill, T. W., A. M. Rymer, J. L. Burch, F. J. Crary, D. T. Young, M. F. Thomsen, D. Delapp, N. André, A. J. Coates, and G. R. Lewis (2005), Evidence for rotationally driven plasma transport in Saturn's magnetosphere, Geophys. Res. Lett., 32, L14S10, doi:10.1029/2005GL022620.

\section{Introduction}

[2] Radial transport of plasma in a rotation-dominated magnetosphere is expected to occur through alternating longitudinal sectors of cooler, denser plasma moving outward and hotter, more tenuous plasma moving inward [e.g., Pontius et al., 1986; Yang et al., 1994]. An observable consequence of the inward $\mathbf{E} \times \mathbf{B}$ transport of hot plasma is longitudinal drift dispersion of the injected plasma (Figure 1), forming a $\mathrm{V}$ shape in a linear energy versus longitude spectrogram (Figure 2). The V shape results from the adiabatic gradient and curvature drifts, proportional to \footnotetext{
USA.

${ }^{1}$ Physics and Astronomy Department, Rice University, Houston, Texas,

${ }^{2}$ Mullard Space Science Laboratory, University College London, Surrey, UK.

${ }^{3}$ Space Science and Engineering Division, Southwest Research Institute, San Antonio, Texas, USA.

${ }^{4}$ Los Alamos National Laboratory, Los Alamos, New Mexico, USA.

${ }^{5}$ Centre d'Etude Spatiale des Rayonnements, Toulouse, France.
}

Copyright 2005 by the American Geophysical Union. 0094-8276/05/2005GL022620 particle energy and to charge sign. These injection/dispersion signatures are observed frequently by geosynchronous spacecraft in Earth's magnetosphere [e.g., Deforest and McIlwain, 1971] and less frequently by the Galileo spacecraft in Jupiter's [Mauk et al., 1997, 1999].

[3] Saturn's magnetosphere turns out to be an ideal laboratory for studying these signatures. Localized hotplasma injections are numerous and often isolated, and the $\mathrm{V}$ structures are swept past a spacecraft by the prevailing partial corotational flow of the ambient plasma, which is much faster than both the gradient-curvature drift speed and the motion of the spacecraft relative to Saturn. The energylongitude $\mathrm{V}$ structures are thus transformed into directly observable energy-time V structures. The drift dispersion is much more prominent at Saturn than at Jupiter because the gradient-curvature drift speed is faster, for a given particle energy and dipole $L$ value, by a factor $\left(\mathrm{B}_{\mathrm{J}} \mathrm{R}_{\mathrm{J}} / \mathrm{B}_{\mathrm{S}} \mathrm{R}_{\mathrm{S}}\right) \approx 23$, where $B_{J, S}$ is surface equatorial dipole field strength and $\mathrm{R}_{\mathrm{J}, \mathrm{S}}$ is planetary radius. This difference largely explains the fact that similar signatures at Jupiter are relatively rare, even though the underlying radial transport process is probably even more vigorous at Jupiter than at Saturn.

[4] During its first two orbits of Saturn, the Cassini Plasma Spectrometer [Young et al., 2004] observed 100 such events. An exact count is not feasible because there is a fuzzy line between events and non-events. For this study we have selected a subset of 48 injection/dispersion events that were sufficiently isolated to be easily identified, and sufficiently pronounced to permit a plausible estimate of the slope and thickness of the legs of the V, from which the age and longitudinal width of the injection event can be deduced. These events are clustered in the range $5<L<10$ where $L=R /\left(\mathrm{R}_{\mathrm{S}} \cos ^{2} \lambda\right)$ is the dipole $L$ value $(R=$ planetcentered distance, $\mathrm{R}_{\mathrm{S}}=$ Saturn's radius $\approx 60,300 \mathrm{~km}$, and $\lambda=$ magnetic latitude). Of these 48 events, 12 were observed on the initial ("Rev 0") orbit and 36 on the second ("Rev A") orbit. This occurrence distribution is consistent with the slower radial spacecraft velocity on Rev A, which not only increases the amount of time spent in the relevant radial range (by a factor $>2$ ) but also enhances the probability of identifying a structure that is extended in longitude but restricted in radial distance.

\section{Injection/Dispersion Signatures}

[5] Several examples are evident in the two-hour time interval shown in Figure 2, from the inbound leg of Rev A. The color panels are electron (top) and positive ion (bottom) count-rate spectrograms, from the Electron Spectrometer (ELS) and from the Ion Mass Spectrometer (IMS, summed over detection angles), respectively. Count rate is roughly 


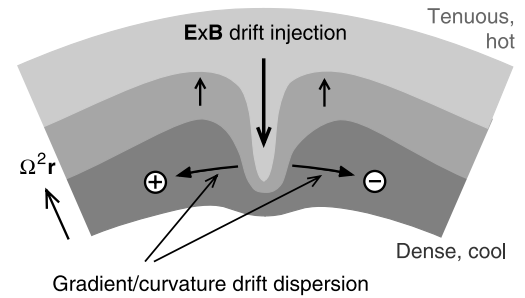

Figure 1. Schematic illustration of a localized injection of hot plasma accompanied by adiabatic gradient and curvature drift, producing a V-shaped dispersion signature on a linear energy-longitude spectrogram.

proportional to energy flux per unit energy interval. Instead of the conventional $\log$ (energy) scale we show a linear energy scale for which the expected drift dispersion signature is a pair of straight lines intersecting on the zero-energy axis to form a "V". For the magnetic polarity of Saturn (like Jupiter's but opposite Earth's), positive ions form the left half of the $\mathrm{V}$ and electrons the right half. Roughly half of the identifiable electron dispersion signatures have visible ion partners. As is typical for a given plasma density, electron count rates are much higher than ion count rates. When visible, the ion signature always has the expected relation to the electron signature, completing the symmetrical V. There is a small asymmetry because the ion drift adds to corotation while the electron drift subtracts; this asymmetry is a few percent or less below $50 \mathrm{keV}$.

[6] Beneath the spectrograms we show the perturbation of the magnetic-field magnitude observed simultaneously by the Cassini MAG experiment, after subtracting a cubic fit to the larger-scale variation. The particle dispersion signatures are, in most cases, accompanied by localized diamagnetic depressions of $|\mathbf{B}|$ essentially coincident with the apex of the V. There is also sometimes an independently observed density cavity in the cooler background plasma near the apex of the V [Burch et al., 2005, Figure 1]. It appears that the older events are less likely to retain their associated diamagnetic depressions and density cavities, although further work on this question is needed. We return to this point below.

[7] Mauk et al. [2005] report similar dispersion signatures observed at higher energies with the Cassini MIMI experiment. They show that the slope of the dispersion curve may be affected in magnitude, and even reversed in sign for sufficiently old injections, by the radial spacecraft motion coupled with the shearing effect of a partial corotation rate that decreases with distance. This effect introduces additional uncertainty into our age estimates reported below, particularly for the oldest events. There are, indeed, a few CAPS electron events on 1 July 2004, 0700-0800 that have a reverse slope of the type reported by Mauk et al., and in the same general region. These exceptional events are not included in our statistical sample, but warrant further study. An interesting outstanding question is whether a subset of the events reported here can be matched in a continuous fashion with the higher-energy events reported by Mauk et al.

[8] Alternative ways to show the energy-time dispersion signature (not shown here) are to plot intensity versus time at several energies [Mauk et al., 1997, Figure 2] or intensity versus energy at several times [Burch et al., 2005, Figure 3].

\section{Analysis}

[9] Each event selected for further analysis has an identifiable energy-time slope, an identifiable center time (apex of the V), and an identifiable width for each leg of the V. These are the selection criteria. Some obvious injection events were excluded from our sample because their slopes were too large to measure reliably; examples of these exceptionally young events are presented and discussed by Burch et al. [2005].

[10] The energy-time slope $d E / d t$ is related to the age of the event since injection $\left(T_{i n j}\right)$ by

$$
d E / d t=(-6.9 \mathrm{keV} / \mathrm{min})(10 / L)\left(1 \mathrm{hr} / T_{i n j}\right)\left(\Omega / \Omega_{\mathrm{S}}\right) \operatorname{Sign}(q)
$$

where $\Omega$ is the angular rate at which the drift dispersion structure is swept past the spacecraft by the partially corotating flow, and $\Omega_{\mathrm{S}} \approx 2 \pi / 10.8 \mathrm{hr}$ is Saturn's rotation frequency. The ages reported below are based on the assumption $\Omega=\Omega_{\mathrm{S}}$, which is an overestimate of $\Omega$ (and hence of $T_{i n j}$ ) by as much as a few tens of percent [Eviatar and Richardson, 1986; Thomsen et al., 2004]. More precise values for $\Omega$ will be available in the future pending further analysis of the ion velocity distributions observed by CAPS. Equation (1) assumes that particles of all energies are injected at a certain longitude at a certain time, and subsequently drift according to the standard gradient and curvature drift formulas in a dipole magnetic field with

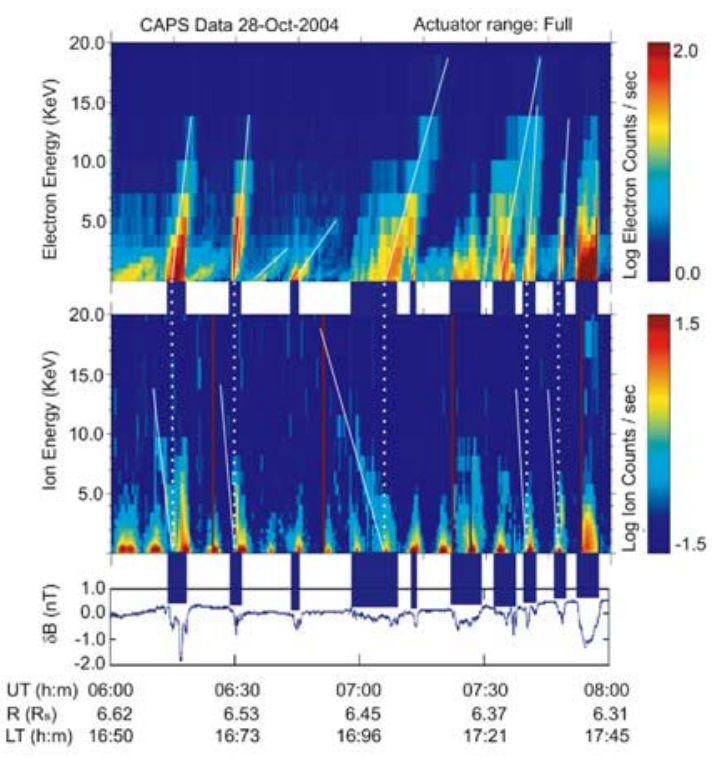

Figure 2. Linear energy-time spectrograms for electrons (top) and positive ions (middle) from the CAPS instruments on 28 October 2004 during the second Cassini orbit of Saturn. The bottom panel shows simultaneous magneticfield magnitude perturbations from the MAG instrument, after subtracting a cubic polynomial fit to the larger-scale variation. The beaded appearance of the ion spectrogram at low energies is an artifact produced by rotation of the instrument. 

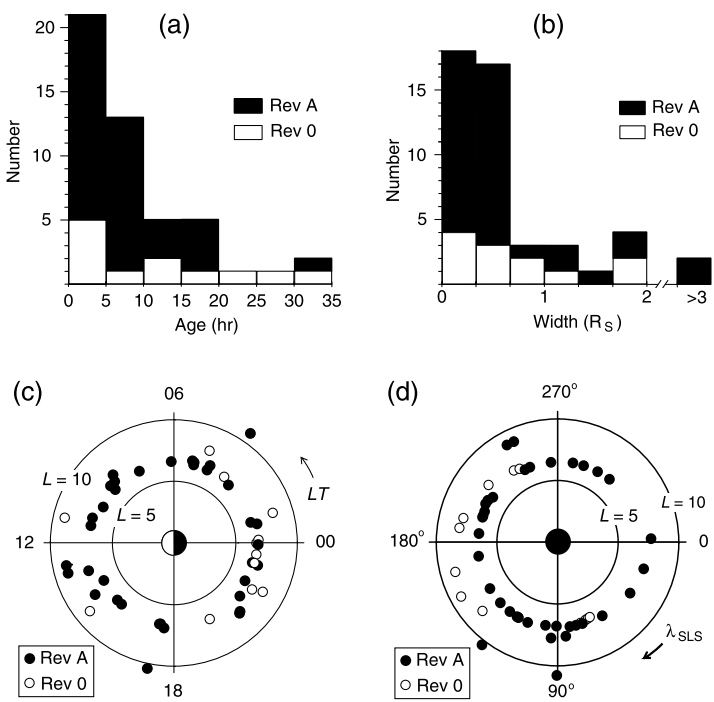

Figure 3. Statistical properties of the 48 injection events selected from the first two Cassini orbits of Saturn. (a) Age since injection, (b) azimuthal width, (c) local time at injection, (d) SLS longitude at injection. In (c) and (d), the radial coordinate is the $\mathrm{L}$ value of observation. All properties are based on the assumption $\Omega=\Omega_{\mathrm{S}}$ (see text).

moment 0.21 G-R $\mathrm{R}_{\mathrm{S}}^{3}$. Equation (1) is for equatoriallymirroring particles, but is adequate within a factor of order unity for particles of any pitch angle.

[11] The width $\delta t$ (in time) of each leg of the $\mathrm{V}$ is proportional to the width $\delta \lambda_{\text {SLS }}$ (in longitude) of the injection feature that produced it: $\delta \lambda_{\mathrm{SLS}} \approx \Omega \delta t$, where SLS denotes the (corotating) Saturn longitude system. Ideally the width $\delta t$ should be (and roughly is) independent of energy for a given structure, and should remain constant in time as the structure rotates (a feature that cannot be tested because we see each structure only once). Assuming $\Omega=\Omega_{\mathrm{S}}$ and multiplying by $L$ we obtain the widths (in $\mathrm{R}_{\mathrm{S}}$ ) reported below.

[12] If $\Omega=\Omega_{\mathrm{S}}$, the SLS longitude of injection is the same as that of the observation. We provisionally assume $\Omega=\Omega_{\mathrm{S}}$ below in reporting the longitudes of injection; thus, we overestimate the injection longitude by the unknown amount $\Delta \lambda_{\mathrm{SLS}}=\left(\Omega_{\mathrm{S}}-\Omega\right) T_{i n j}$. $\left(\lambda_{\mathrm{SLS}}\right.$ is a left-handed angle about the spin axis.) The local time of injection LT(inj) is related to that of the observation $\mathrm{LT}(o b s)$ by $\mathrm{LT}(o b s)=$ $\mathrm{LT}(i n j)+\Omega T_{i n j}$. Again we provisionally assume $\Omega=\Omega_{\mathrm{S}}$ and thereby underestimate LT(inj) by the same unknown amount $\Delta \mathrm{LT}(i n j)=\left(\Omega_{\mathrm{S}}-\Omega\right) T_{i n j}$. (LT is a right-handed angle.)

\section{Results}

[13] Figure 3 shows statistical distributions of (a) the ages, (b) the longitudinal widths, (c) the injection local times, and (d) the injection longitudes of the 48 events selected for study.

[14] Ages (Figure 3a) range from $\sim 1 / 2 \mathrm{hr}$ to several Saturn rotation periods (tens of hr). The lower limit is essentially the smallest age that passes our selection criteria; younger events are observed [Burch et al., 2005] but are indistinguishable from "spikes" with vertical slope. The upper limit is also no doubt a selection effect: the injection/ dispersion structure becomes less distinct, and more likely to overlap neighboring structures, as it gets older. Probably the most significant feature of the age distribution is its lack of features, i.e., the lack of any obvious periodicity in the range of an hour to a few tens of hours.

[15] Longitudinal widths (Figure $3 b$ ) range from $\sim 0.1 R_{S}$ to several $\mathrm{R}_{\mathrm{S}}$. Again, the lower limit is no doubt a selection effect: narrower structures [e.g., Burch et al., 2005] would not have been clearly identified as dispersion structures. The upper limit may be significant, tending to rule out globalscale (many $\mathrm{R}_{\mathrm{S}}$ ) structures. The injections are evidently "mesoscale" structures, much smaller than the magnetosphere but much larger than the ion gyroradius $\left(\sim 0.035 \mathrm{R}_{\mathrm{S}}\right.$ for a $5-\mathrm{keV} \mathrm{O}^{+}$ion at $L=10$ ).

[16] Local times of injection (Figure 3c) are distributed as randomly as could be expected for a sample of this size. There is no obvious preference for a particular LT sector. Longitudes of injection (Figure 3d) are also fairly randomly distributed, although they tend to favor longitudes near $\lambda_{\text {SLS }}=190^{\circ}$. There is no obvious orbital factor that would produce this asymmetry. It will be interesting to see if this asymmetry persists on future orbits.

[17] Measurement error is negligible compared to errors introduced by our analysis. Our assumption $\Omega=\Omega_{\mathrm{S}}$, as noted above, causes us to overestimate the ages and longitudes, and underestimate the widths and local times, of individual injection events, by perhaps a few tens of percent. By comparison, the dipole-field assumption is probably less of a problem because the outward stretching of the magnetic field tends to increase the curvature-drift speed at the equator while decreasing the gradient-drift speed, so that their sum varies less than either one separately.

[18] The other significant error source is simply the uncertainty in reading observable properties from the spectrograms. Reading the center time, slope, and width for a given event is a necessarily subjective process. The associated random errors are estimated to be $\sim \pm 1$ min for center times (or less for narrower structures), $\pm 20 \%$ for slopes, and $\pm 10 \%$ for widths. The ability to achieve this level of accuracy was indeed the main selection criterion. This is why some events that are evident in Figure 2 were excluded from the statistical sample.

\section{Implications}

[19] The results reported here are consistent with the hypothesis that radial plasma transport in Saturn's magnetosphere is accomplished by mesoscale convection cells driven by the centrifugal interchange instability. The radial distribution of flux-shell mass content is energetically unstable to centrifugal interchange [Eviatar and Richardson, 1986; Thomsen et al., 2004], and the isolated injection structures inferred here are an expected consequence of this instability [e.g., Pontius et al., 1986; Yang et al., 1994]. The lack of a local-time asymmetry (Figure 3c) argues for a rotationally driven transport process rather than a solar-wind-driven process as at Earth, where such signatures are largely restricted to the night side and therefore attributed to substorm injection from the magnetotail. 
[20] Additional work is needed to determine the conditions for the existence of a density cavity and/or diamagnetic disturbance at the center of a given injection/ dispersion event. If a given flux tube were to be transported adiabatically outward, and then back inward to the same radius, it would return with the same density and temperature with which it left, neglecting chemical sources and losses during the transport. This can be taken as the definition of adiabatic transport. The fact that the injected flux tubes have much lower density and much higher temperature than the background indicates that they have suffered a non-adiabatic loss of density and a non-adiabatic gain of temperature during the cycle of outward/inward transport. The most obvious way to achieve both of these changes at once is to suppose that the flux tube is blown open in a downstream planetary wind [Hill et al., 1974; Vasyliunas, 1983] between the outward and inward halves of the transport cycle. This process automatically reduces density, by losing particles, and simultaneously increases temperature, by the heating that accompanies the implied reconnection [Carbary et al., 1976].

[21] In any case, the non-adiabatic temperature gain evidently outweighs the non-adiabatic density loss, because the injected flux tubes typically contain higher plasma pressure than their surroundings. This is evidenced by the fact that the diamagnetic disturbance is typically, though not always, negative (Figure 2). The diamagnetic disturbance is sometimes absent, for example in the two older signatures that are visible between 0600 and 0630 in Figure 2 but not included in the statistical sample. Occasionally (not shown here) the diamagnetic disturbance is even positive, indicating a plasma pressure deficit.

[22] With additional orbits and additional analysis, CAPS will produce characteristic radial profiles of plasma densities and temperatures for electrons, protons, and watergroup ions. It will then become possible to infer the radial distance from which a given injection structure originated, following the earlier analysis of Galileo data at Jupiter [Bolton et al., 1997; Kivelson et al., 1997; Thorne et al., 1997]. It will also become possible to estimate the rate at which plasma mass, and hence energy [Hill et al., 1983], are transported outward from internal sources.

[23] Acknowledgments. We have benefited from conversations with A. J. Dessler, B. H. Mauk, and C. T. Russell. The work of U.S. co-authors was supported by NASA/JPL under contract 1243218 with SwRI. Work at MSSL was supported by the Particle Physics and Astronomy Research Council, and at CESR by the French space agency CNES. Work at Los Alamos was performed under the auspices of the U.S. Department of Energy.

\section{References}

Bolton, S. J., R. M. Thorne, D. A. Gurnett, W. S. Kurth, and D. J. Williams (1997), Enhanced whistler-mode emissions: Signatures of interchange motion in the Io plasma torus, Geophys. Res. Lett., 24, 2123.

Burch, J. L., J. Goldstein, T. W. Hill, D. T. Young, F. J. Crary, A. J. Coates, N. André, W. S. Kurth, and E. C. Sittler Jr. (2005), Properties of local plasma injections in Saturn's magnetosphere, Geophys. Res. Lett., 32, L14S02, doi:10.1029/2005GL022611.

Carbary, J. F., T. W. Hill, and A. J. Dessler (1976), Planetary-spin period acceleration of particles in the Jovian magnetosphere, J. Geophys. Res., 81,5189 .

DeForest, S. E., and C. E. McIlwain (1971), Plasma clouds in the magnetosphere, J. Geophys. Res., 76, 3587.

Eviatar, A., and J. D. Richardson (1986), Corotation of the Kronian magnetosphere, J. Geophys. Res., 91, 3299.

Hill, T. W., A. J. Dessler, and F. C. Michel (1974), Configuration of the Jovian magnetosphere, Geophys. Res. Lett., 1, 3.

Hill, T. W., A. J. Dessler, and C. K. Goertz (1983), Magnetospheric models, in Physics of the Jovian Magnetosphere, edited by A. J. Dessler, chap. 10, pp. 353-394, Cambridge Univ. Press, New York.

Kivelson, M. G., K. K. Khurana, C. T. Russell, and R. J. Walker (1997), Intermittent short-duration plasma-field anomalies in the Io plasma torus: Evidence for interchange in the Io plasma torus, Geophys. Res. Lett., 24, 2127.

Mauk, B. H., D. J. Williams, and R. W. McEntire (1997), Energy-time dispersed charged particle signatures of dynamic injections in Jupiter's inner magnetosphere, Geophys. Res. Lett., 24, 2949.

Mauk, B. H., et al. (1999), Storm-like dynamics of Jupiter's inner magnetosphere, J. Geophys. Res., 104, 22,759.

Mauk, B. H., et al. (2005), Energetic particle injections in Saturn's magnetosphere, Geophys. Res. Lett., 32, L14S05, doi:10.1029/2005GL022485.

Pontius, D. H., Jr., T. W. Hill, and M. E. Rassbach (1986), Steady state plasma transport in a corotation-dominated magnetosphere, Geophys. Res. Lett., 13, 1097.

Thomsen, M. F., et al. (2004), Transport in Saturn's outer magnetosphere: Cassini observations, Eos Trans. AGU, 85(47), Fall Meet. Suppl., Abstract P53B-05.

Thorne, R. M., T. P. Armstrong, S. Stone, D. J. Williams, R. W. McEntire, S. J. Bolton, D. A. Gurnett, and M. G. Kivelson (1997), Galileo evidence for rapid inward transport in the Io torus, Geophys. Res. Lett., 24, 2131.

Vasyliunas, V. M. (1983), Plasma distribution and flow, in Physics of the Jovian Magnetosphere, edited by A. J. Dessler, chap. 11, pp. 395-453, Cambridge Univ. Press, New York.

Yang, Y. S., R. A. Wolf, R. W. Spiro, T. W. Hill, and A. J. Dessler (1994), Numerical simulation of torus-driven plasma transport in the Jovian magnetosphere, J. Geophys. Res., 99, 8755.

Young, D. T., et al. (2004), Cassini Plasma Spectrometer investigation, Space Sci. Rev., 114, 1-112.

N. André, Centre d'Etude Spatiale des Rayonnements, F-31028 Toulouse, France.

J. L. Burch, F. J. Crary, and D. T. Young, Space Science and Engineering Division, Southwest Research Institute, San Antonio, TX 78228, USA.

A. J. Coates, G. R. Lewis, and A. M. Rymer, Mullard Space Science Laboratory, University College London, Surrey RH5 6NT, UK.

D. Delapp and M. F. Thomsen, Los Alamos National Laboratory, Los Alamos, NM 87545, USA.

T. W. Hill, Physics and Astronomy Department, Rice University, Houston, TX 77005, USA. (hill@rice.edu) 